\title{
Single nucleotide polymorphism of MTHFR rs1801133 associated with elevated Hcy levels affects susceptibility to cerebral small vessel disease
}

\author{
Hongyu Yuan ${ }^{1}$, Man Fu ${ }^{2}$, Xianzhang Yang ${ }^{1}$, Kun Huang ${ }^{1}$, Xiaoyan Ren ${ }^{\text {Corresp. } 1}$ \\ ${ }^{1}$ The Third Department of Neurology, Heze Municipal Hospital, Heze, Shandong Province, China \\ 2 Department of Neurology, The Third People's Hospital of Heze, Heze, Shandong Province, China \\ Corresponding Author: Xiaoyan Ren \\ Email address: ren_1667@sina.com
}

Background. Methylenetetrahydrofolate reductase (MTHFR) is indispensable for the conversion of homocysteine ( $\mathrm{Hcy}$ ) to methionine. The single nucleotide polymorphism (SNP) of MTHFR gene ( $r$ 1801133, C667T) is correlated with decreased enzyme activity that eventually results in elevated plasma Hcy levels. Hyperhomocysteinemia has been confirmed to be involved in the pathogenesis of stroke, cerebral small vessel disease (CSVD), various metabolic disorders and so on. However, the relationship between the MTHFR gene polymorphisms, Hcy, and CSVD has not been investigated. In this study, the relationship between SNPS of MTHFR gene and CSVD was determined after adjusting for cardiovascular risk factors, and the potential mechanism based on Hcy levels was explored. Methods. A total of 163 consecutive CSVD patients were collected as the case group. In the corresponding period, 326 healthy people were selected as the control group, who were matched to these cases according to age ( \pm 2 years) and gender at a ratio of 2:1. SNPs of MTHFR rs1801133, rs1801131, rs2274976, rs4846048, rs4846049, rs13306561 and rs3737964 were genotyped with TaqMan Pre-Designed SNP Genotyping Assays. Plasma Hcy levels were detected using Hcy reagent through enzymatic cycling assay. Multivariate analysis was used to identify the SNPs associated with CSVD susceptibility. Plasma Hcy levels were compared between different genotypes. Results. The MTHFR rs1801133 TT and CT genotype had increased risk for CSVD, and the OR was higher in the $\Pi$ genotype than in the CT genotype (2.307 vs 1.473). The plasma Hcy levels of different genotypes showed the tendency of the TT genotype >CT genotype >CC genotype ( $19.91 \pm 8.73 \mathrm{pg} / \mathrm{ml}$ vs $17.04 \pm 5.68 \mathrm{pg} / \mathrm{ml}$ vs $14.96 \pm 4.85 \mathrm{pg} / \mathrm{ml})$. Conclusions. The SNP of MTHFR rs1801133 was correlated with CSVD, and the TT and CT genotypes had increased risk for CSVD compared to the CC genotype. The potential mechanism was associated with elevated Hcy levels. 
1 Single nucleotide polymorphism of MTHFR rs1801133 associated with elevated Hcy levels

2 affects susceptibility to cerebral small vessel disease

3 Hongyu Yuan ${ }^{1}$, Man Fu², Xianzhang Yang ${ }^{1}$, Kun Huang ${ }^{1}$, Xiaoyan Ren ${ }^{1}$

4 1. The Third Department of Neurology, Heze Municipal Hospital, Heze 274031, China

5 2. Department of Neurology, The Third People's Hospital of Heze, Heze 274031, China

6 Address correspondence to:

$7 \quad$ Xiaoyan Ren

8 The Third Department of Neurology, Heze Municipal Hospital

9 No. 2888, Caozhou West Road, Mudan District, Heze 274031, China

10 Tel: $+86-5305265053$

11 Fax:+86-5305613311

12 Email: ren_1667@sina.com 


\section{Abstract}

Background. Methylenetetrahydrofolate reductase (MTHFR) is indispensable for the conversion of homocysteine (Hcy) to methionine. The single nucleotide polymorphism (SNP) of MTHFR gene (rs1801133, C667T) is correlated with decreased enzyme activity that eventually results in elevated plasma Hcy levels. Hyperhomocysteinemia has been confirmed to be involved in the pathogenesis of stroke, cerebral small vessel disease (CSVD), various metabolic disorders and so on. However, the relationship between the MTHFR gene polymorphisms, Hcy, and CSVD has not been investigated. In this study, the relationship between SNPs of MTHFR gene and CSVD was determined after adjusting for cardiovascular risk factors, and the potential mechanism based on Hcy levels was explored.

Methods. A total of 163 consecutive CSVD patients were collected as the case group. In the corresponding period, 326 healthy people were selected as the control group, who were matched to these cases according to age ( \pm 2 years) and gender at a ratio of $2: 1$. SNPs of MTHFR rs1801133, rs1801131, rs2274976, rs4846048, rs4846049, rs13306561 and rs3737964 were genotyped with TaqMan Pre-Designed SNP Genotyping Assays. Plasma Hcy levels were 
37 detected using Hcy reagent through enzymatic cycling assay. Multivariate analysis was used to

38 identify the SNPs associated with CSVD susceptibility. Plasma Hcy levels were compared

39 between different genotypes.

40 Results. The MTHFR rs1801133 TT and CT genotype had increased risk for CSVD, and the $O R$

41 was higher in the TT genotype than in the CT genotype (2.307 vs 1.473$)$. The plasma Hcy levels

42 of different genotypes showed the tendency of the TT genotype $>$ CT genotype $>$ CC genotype

$43(19.91 \pm 8.73 \mathrm{pg} / \mathrm{ml}$ vs $17.04 \pm 5.68 \mathrm{pg} / \mathrm{ml}$ vs $14.96 \pm 4.85 \mathrm{pg} / \mathrm{ml})$.

44 Conclusions. The SNP of MTHFR rs1801133 was correlated with CSVD, and the TT and CT

45 genotypes had increased risk for CSVD compared to the CC genotype. The potential mechanism

46 was associated with elevated Hcy levels.

47 Key words: Methylenetetrahydrofolate reductase; Single nucleotide polymorphism;

48 Homocysteine; Cerebral small vessel disease

\section{Introduction}

51 As a generic term for intracranial vascular disease associated with various neurological and

52 pathological processes, cerebral small vessel disease (CSVD) refers to a syndrome of different

53 clinical features and neuroimaging findings induced by pathological changes in capillaries,

54 perforating cerebral arterioles and venules (Shi and Wardlaw, 2016). CSVD is the cause of $45 \%$

55 of all the cases of dementia, and accounts for $25 \%$ of the ischemic stroke cases around the world 
56 (Pantoni, 2010; Petty et al., 2000; Wardlaw et al., 2013). Additionally, CSVD is a major cause

57 for depression, cognitive impairment, disability and so on in the aged (Li et al., 2018).

58 The pathogenesis for CSVD mainly includes impairment of cerebral autoregulation, reduction of

59 cerebral blood flow and increase of blood-brain barrier (BBB) permeability (Joutel and

60 Chabriat, 2017; Li et al., 2018; Li et al., 2019). However, its molecular mechanisms are not

61 completely elucidated. Genetic studies have demonstrated that CSVD is highly heritable,

62 especially in young-onset stroke patients, and that disease processes of some CSVD subtypes

63 may be associated with common variants in monogenic disease genes (Tan et al., 2017).

64 Methylenetetrahydrofolate reductase (MTHFR) gene is located on chromosome 1p36.3, and it is

65 associated with the biosynthesis pathway of amino acid and purine (Goyette et al., 1994; Cui et

66 al., 2011). As an important regulatory enzyme catalyzing the transformation of 5, 10-

67 methylenetetrahydrofolate to 5-methyltetrahydrofolate, MTHFR is indispensable for the

68 conversion of homocysteine (Hcy) to methionine (Qin et al., 2012; Pogliani et al., 2015). The

69 single nucleotide polymorphism (SNP) of MTHFR gene (rs1801133, C667T) is correlated with

70 decreased enzyme activity that eventually results in elevated plasma homocysteine levels

71 (Engbersen et al., 1995; Kang et al., 1993). Hyperhomocysteinemia has been confirmed to be

72 involved in the pathogenesis of stroke, CSVD, various metabolic disorders and so on (Inamoto et

73 al., 2003; Zee et al., 2007; Chutinet et al., 2012; Qin et al., 2017; Nam et al., 2019; Piao et al.,

74 2018; Kloppenborg et al., 2011; Jeon et al., 2014; Pavlovic et al., 2011). In addition, a recent

75 study suggested that MTHFR C677T genotype was associated with CSVD subtype (Rutten- 
76 Jacobs et al., 2016). However, no previous studies have investigated the mechanism associated

77 with the effect of MTHFR C677T genotype on CSVD susceptibility. In this study, the relationship between SNPs of MTHFR gene (rs1801133, rs1801131, rs2274976, rs4846048, rs4846049, rs13306561 and rs3737964) and CSVD was determined after adjusting for cardiovascular risk factors, and the potential mechanism based on Hcy levels was explored. The aim was to provide useful clues for identifying susceptible populations of CSVD.

\section{Materials \& Methods}

\section{Participants}

85 A total of 163 consecutive patients with CSVD were collected as the case group in Heze

86 Municipal Hospital between April 2017 and October 2018. In the corresponding period, 326

87 healthy people were selected as the control group, who were matched to these cases according to

88 age ( \pm 2 years) and gender at a ratio of $2: 1$. This study received the permission of the ethic

89 committee of Heze Municipal Hospital (20160141022), and written informed consent was

90 provided by each participant.

91 The inclusion criteria of CSVD patients were (1) in accordance with the diagnostic criteria

92 suggested by Shi Y and Wardlaw JM (Shi and Wardlaw, 2016); (2) to demonstrate typical

93 neuroimaging changes in the subcortical grey matter and white matter, including white matter

94 hyperintensities (WMHs), prominent perivascular spaces (PVS), cerebral microbleeds (CMBs), 
95

96

97

98

99

100

101

102

103

104

105

106

107

108

109

110

111

112

113

atrophy, lacunas and recent small subcortical infarct (Wardlaw et al., 2013); (3) complete clinical

data; (4) written informed consent. The exclusion criteria included (1) large-artery

atherosclerosis; (2) a definite history of subarachnoid hemorrhage or cerebral hemorrhage; (3)

acute ischemic stroke caused by cardiogenic embolism.

\section{Data Collection}

Demographic data, cardiovascular risk factors and laboratory indexes were collected in each participant. Demographic data included age, gender, height, weight, annual family income, education level and occupation. Cardiovascular risk factors included hypertension, hyperlipemia, diabetes, smoking, drinking and blood pressure. Laboratory indexes included total cholesterol, triacylglycerol (TG), low density lipoprotein cholesterol (LDL-C), high density lipoprotein cholesterol (HDL-C) and fasting blood glucose (FBG).

\section{SNP genotyping for MTHFR gene}

The DNA used in SNP genotyping was extracted from peripheral blood using the salting out method suggested by Hashemi et al (Hashemi et al., 2010). TaqMan Pre-Designed SNP Genotyping Assays (Applied Biosystems, Carlsbad, USA) was employed to perform SNP genotyping for the MTHFR gene (rs1801133, rs1801131, rs2274976, rs4846048, rs3737966, rs 1537515, rs4846049, rs3834044, rs13306561 and rs3737964). The ABI 7500 Fast real-time PCR system (Applied Biosystems, Carlsbad, USA) was employed to perform PCR amplification and allelic discrimination. 


\section{Plasma Hcy assay}

115 The blood sample was collected from the antecubital vein after an overnight fasting in each

116 participant. The blood sample was then separated through centrifugation at $3000 \mathrm{~g}$ for $5 \mathrm{~min}$.

117 The level of Hcy was detected by enzymatic cycling assay using Hcy reagent (Beijing Strong

118 Biotechnologies, Inc, Beijing, China) and the ROCHE Cobas 8000 automatic biochemical

119 analyzer (Roche Ltd., Switzerland) .

\section{Statistical analysis}

121 Haploview software version 4.2 (http://www.broad.mit.edu/mpg/haploview; developed in Mark

Daly's laboratory at the Broad Institute) was employed to perform Hardy-Weinberg equilibrium

123 (HWE) test and to calculate allele frequencies and genotype frequencies for all the SNPs (Barrett,

124 2009). Univariate analysis was performed with Student's $t$-test or chi-square test for all variables,

125 including demographic data, vascular risk factors, laboratory indexes and SNP genotyping data.

126 The variables with a $P$ value less than 0.10 were then included in the multivariate analysis, which

127 was used to identify the SNPs associated with CSVD susceptibility through a backward stepwise

128 logistic regression model. Plasma Hcy levels among different genotypes were compared using

129 ANOVA. All statistical analysis was conducted using the SPSS version 20.0 for Windows (SPSS

130 Inc., USA), and significance was set at $P<0.05$. 
133

134

135

136

137

138

139

140

141

142

143

144

145

146

147

148

149

150

151

\section{Univariate analysis of demographic data, vascular risk factors and laboratory indexes}

The case group included 94 males and 69 females, and the control group included 188 males and 138 females. Their average age was (63.28 \pm 7.09$)$ years. Univariate analysis demonstrated that age, body mass index (BMI), annual family income, education level, occupation, drinking, hyperlipidaemia, total cholesterol, TG, LDL-C and HDL-C were not statistically different between the case group and the control group, and hypertension, diabetes, smoking, systolic blood pressure (SBP), diastolic blood pressure (DBP) and FBG were statistically different (Table 1).

\section{SNP analysis}

All the SNPs were successfully genotyped in both the case group and the control group. As shown in Table 2, the genotype frequencies of all the SNPs were not statistically different from those evaluated using Hardy-Weinberg equilibrium. Univariate analysis demonstrated that the genotype frequencies of $\operatorname{rs} 1801133\left(\chi^{2}=12.852, P=0.002\right)$ and $\operatorname{rs} 1801131\left(\chi^{2}=6.203, P=0.045\right)$

were statistically different between the case group and the control group, and rs2274976, rs4846048, rs4846049, rs13306561 and rs3737964 were not statistically different (all $P>0.05$ ).

\section{Multivariate analysis}

Multivariate analysis was performed to identify the independent association between different genotypes of the MTHFR rs1801133 and rs1801131 and CSVD. The results demonstrated that the polymorphism of rs 1801133 was correlated with CSVD after adjusting hypertension, 
152 diabetes, smoking, SBP, DBP, FBG, hyperlipidaemia, LDL-C and HDL-C, but rs 1801131 was

153 not (Table 3). The MTHFR rs1801133 TT and CT genotype had increased risk for CSVD, and

154 the $O R$ was higher in the TT genotype than in the CT genotype (2.307 vs 1.473).

155

156

157

158

159

160

161

162

163

164

165

166

167

168

169

170

\section{Plasma Hcy levels}

Plasma Hcy levels were compared using ANOVA between the MTHFR rs1801133 TT, CT and CC genotype. The results demonstrated that plasma Hcy levels were highest in the TT genotype, intermediate in the CT genotype, and lowest in the CC genotype (Table 4).

Joint effect of MTHFR 677C $>$ T (rs1801133) and 1298A $>C$ (rs1801131) on plasma Hcy

levels

The combination of genotypes $677 \mathrm{TT}$ and $1298 \mathrm{CC}$ was abbreviated TTCC, 677TT and 1298AA was abbreviated TTAA, etc. The frequencies of the nine combination genotypes derived from the both polymorphisms were TTAA $(35.17 \%, 172 / 489)$, CTAA $(24.34 \%, 119 / 489)$, CTAC $(12.68 \%$, 62/489), CCAC (11.25\%, 55/489), CCAA (8.90\%, 44/489), CCCC (7.16\%, 35/489), CTCC $(0.20 \%, 1 / 489), \quad$ TTAC $(0.20 \%, 1 / 489)$ and TTTC $(0,0 \%)$ in all participants.

The distribution of the six common combination genotypes was significantly different between the case group and the control group (Table 5). Plasma Hcy levels were highest in TTAA genotype, and moderate in CTAA and CTAC genotypes, and lowest in CCAC, CCAA and CCCC genotypes. However, the difference was not significant between CTAA and CTAC genotypes and also between CCAC, CCAA and CCCC genotypes (Table 6). 


\section{Discussion}

173 As a sulfur-containing amino acid, Hcy is an important intermediate product for the metabolism

174 of methionine. Hcy has an important role in vascular function (Li et al., 2017). Elevated Hcy

175 levels can predispose vascular smooth muscle cells and endothelial cells to injury, which leads to

176 activation of coagulation factors, expression of plasminogen activator inhibitor, endothelial

177 proliferation and so on (Hainsworth et al., 2016). This further inhibits the expression of

178 thrombomodulin and synthesis of tissue-type plasminogen activator and sulfated heparin,

179 eventually leading to atherogenesis and thrombogenesis through secretion of inflammatory

180 cytokines, platelet aggregation, endoplasmic reticulum stress, and oxidative stress.

181 Studies show that Hyperhomocysteinemia (HHcy) is associated with many diseases, including

182 ischemic stroke (IS), CSVD and various metabolic disorders and so on. Pavlovic et al. showed

183 that elevated total Hcy was correlated with clinical status and severity of white matter changes in

184 symptomatic patients with subcortical small vessel disease (Pavlovic et al., 2011). HHcy has also

185 been a confirmed independent risk factor for IS (Wu et al., 2016; Boysen et al., 2003). Wu et al.

186 demonstrated that high Hcy levels were associated with a greater incidence of acute cerebral

187 infarction among patients with carotid artery plaques (Wu et al., 2016). Ji et al. reported that high

188 Hcy levels were associated with a poor functional outcome, severe neurological impairment and

189 stroke recurrence in large artery atherosclerosis stroke subtype, which confirmed the atherogenic

190 effect of Hcy (Ji et al., 2015). Lu et al. demonstrated that high Hcy levels were correlated with 
191

192

193

194

195

196

197

198

199

200

201

202

203

204

205

206

207

208

209

210

strong plaque enhancement and acute ischemic stroke with adjustment for sex, age, serum creatinine levels and other atherosclerotic risk factors (Lu et al., 2018). Several previous cohort studies also showed that high Hcy levels were correlated with increased risk of IS, including the British Regional Heart Study, the Framingham Study and the Northern Manhattan cohort study (Perry et al., 1995; Bostom et al., 1999; Sacco et al., 2004). As a risk factor of atherosclerosis, high Hcy levels are associated with white matter lesions, lacunar infarcts and cognitive impairment. Kioppenborg et al. (Kloppenborg et al., 2011) found that a higher Hcy level was associated with presence of lacunar infarcts and a higher volume of white matter lesions among patients with symptomatic atherosclerotic disease. Piao et al. (Piao et al., 2018) evaluated the association between Hcy levels and CSVD with the method of meta-analysis. Their results demonstrated that Hcy levels were higher in CSVD patients than in healthy controls. Nam et al. (Nam et al., 2019) found that serum Hcy levels were associated with the presence of cerebral microbleeds, white matter hyperintensity volume enlarged perivascular spaces in a healthy population. In addition, Hcy levels may be correlated with the susceptibility for NAFLD (Hu et al., 2016; Polyzos et al., 2015).

MTHFR is a key controlling enzyme involved in the metabolism of Hcy and folate. It is indispensable for the conversion of homocysteine to methionine through catalyzing the transformation of 5, 10-methylenetetrahydrofolate to 5-methyltetrahydrofolate (Qin et al., 2012; Pogliani et al., 2015). Additionally, it has a role in chromosomal integrity, DNA methylation and maintaining the stability of single- and double-strand DNA (Robien and Ulrich, 2003). It is

Peer) reviewing PDF | (2019:08:40599:2:0:NEW 15 Jan 2020) 
211

212

213

214

215

216

217

218

219

220

221

222

223

224

225

226

227

228

229

230

encoded by the MTHFR gene, which is located on chromosome 1p36.3 (Goyette et al., 1994).

For the MTHFR gene, the cytosine (C) to thymine (T) substitution at position 677 (rs1801133) in

the gene encoding region is the most common SNP. This variation leads to the conversion from alanine to valine at amino acid 222 (Jadavji et al., 2015), and is correlated with decrease of thermal stability of MTHFR and subsequent decrease of enzyme activity (Atadzhanov et al., 2013; Ou et al., 2014). Compared to the CC genotype, the enzyme activity of the CT and TT genotypes is less than $35 \%$ and $70 \%$, respectively (Frosst et al., 1995). The decreased enzyme activity eventually leads to the elevation of Hcy levels (Atadzhanov et al., 2013; Ou et al., 2014). In other words, the CT and TT genotypes are correlated with elevated Hcy levels through reducing the activity of MTHFR. Wang et al. (Wang et al., 2018) found that the SNP of the MTHFR rs1801133 and NAFLD had a potential synergistic effect on elevated Hcy levels. Li et al. (Li et al., 2017) found that the plasma Hcy levels of different genotypes of the MTHFR rs 1801133 showed the tendency of the TT genotype $>$ CT genotype $>$ CC genotype. They concluded that a possible synergistic effect of the MTHFR rs1801133 SNP on plasma Hcy levels increased the risk of IS. In addition, Rutten-Jacobs et al. demonstrated that MTHFR C677T genotype was associated with CSVD subtype (Rutten-Jacobs et al., 2016).

In this study, the SNP of the MTHFR rs1801133 was correlated with CSVD, and the TT and CT genotypes had increased risk for CSVD compared to the CC genotype. Moreover, the overall response $(O R)$ was higher in the TT genotype than in the CT genotype. At the same time, the plasma Hcy levels of different genotypes showed the tendency of the TT genotype $>$ CT genotype 
$>$ CC genotype. Therefore, the SNP of rs1801133 was correlated with CSVD through elevating

232 Hcy levels. Ulvik et al. (Ulvik et al., 2007) demonstrated a strong linkage and functional

233 inference of MTHFR $677 \mathrm{C}>\mathrm{T}$ and $1298 \mathrm{~A}>\mathrm{C}$ polymorphisms through a large-scale

234 epidemiological investigation. In this study, we further investigated the frequencies of

235 combination genotypes of MTHFR $677 \mathrm{C}>\mathrm{T}$ and $1298 \mathrm{~A}>\mathrm{C}$ in the case group and the control

236 group. The results showed that the distribution of the six common combination genotypes was

237 significantly different between the case group and the control group. Lastly, we investigated

238 plasma Hcy levels of the six common combination genotypes. However, the results showed that

239 the difference was not significant between CTAA and CTAC genotypes and also between CCAC,

240 CCAA and CCCC genotypes. The reason might be associated with a small sample size.

241 In this study, the inclusion and exclusion criteria of CSVD patients used in this paper included

242 clinical symptoms, signs and typical neuroimaging changes. Additionally, CSVD patients should

243 have complete clinical data. A ratio of 2:1 was selected for control people : case people with the

244 aim of improving the efficiency of the study through enhancing statistical power. The objective

245 of this study was to evaluate the relationship between SNPs of MTHFR gene and CSVD

246 comprehensively and precisely, and the main limitation was a small sample size. We will

247 investigate the joint effect of MTHFR $677 \mathrm{C}>\mathrm{T}$ and $1298 \mathrm{~A}>\mathrm{C}$ using a larger sample size in the

248 next step. 
251 The SNP of the MTHFR rs1801133 was correlated with CSVD susceptibility through

252 influencing Hcy levels.

253

254 Acknowledgments

255 None.

256

\section{References}

258 Atadzhanov M, Mwaba MH, Mukomena PN, Lakhi S, Rayaprolu S, Ross OA, Meschia JF. 2013.

259 Association of the APOE, MTHFR and ACE genes polymorphisms and stroke in Zambian

260 patients. Neurol Int 5(4): e20. DOI: 10.4081/ni. 2013.e20.

261 Bostom AG, Rosenberg IH, Silbershatz H, Jacques PF, Selhub J, D'Agostino RB, Wilson PW,

262 Wolf PA. 1999. Nonfasting plasma total homocysteine levels and stroke incidence in elderly

263 persons: the Framingham study. Ann Intern Med 131(5):352-355.

Boysen G, Brander T, Christensen H, Gideon R, Truelsen T. 2003. Homocysteine and risk of

265 recurrent stroke. Stroke 34(5):1258-1261.

266

Barrett JC. 2009. Haploview: visualization and analysis of SNP genotype data. Cold Spring Harb Protoc 2009(10):pdb.ip71. DOI: 10.1101/pdb.ip71. 
269 Kim KS. 2011. Methylenetetrahydrofolate reductase C677T polymorphism in patients with lung 270 cancer in a Korean population. BMC Med Genet 12:28. DOI: 10.1186/1471-2350-12-28.

271 Chutinet A, Suwanwela NC, Snabboon T, Chaisinanunkul N, Furie KL, Phanthumchinda K.

272 2012. Association between genetic polymorphisms and sites of cervicocerebral artery

273 atherosclerosis. J Stroke Cerebrovasc Dis 21(5):379-385. DOI:

274 10.1016/j.jstrokecerebrovasdis.2010.10.002.

275 Engbersen AM, Franken DG, Boers GH, Stevens EM, Trijbels FJ, Blom HJ. 1995. Thermolabile 276 5,10-methylenetetrahydrofolate reductase as a cause of mild hyperhomocysteinemia. Am J Hum 277 Genet 56(1):142-150.

278 Frosst P, Blom HJ, Milos R, Goyette P, Sheppard CA, Matthews RG, Boers GJ, den Heijer M, 279 Kluijtmans LA, van den Heuvel LP, Rozen R. 1995. A candidate genetic risk factor for vascular 280 disease: a common mutation in methylenetetrahydrofolate reductase. Nat Genet 10(1):111-113.

281 Goyette P, Sumner JS, Milos R, Duncan AM, Rosenblatt DS, Matthews RG, Rozen R. 1994. 282 Human methylenetetrahydrofolate reductase: isolation of cDNA, mapping and mutation 283 identification. Nat Genet 7(2):195-200. 
288 hyperhomocysteinemia and vascular contributions to cognitive impairment and dementia (VCID). 289 Biochim Biophys Acta 1862(5):1008-1017. DOI: 10.1016/j.bbadis.2015.11.015.

$290 \mathrm{Hu}$ Y, Liu J, Dong X, Xu Y, Leng S, Wang G. 2016. Clinical study of serum homocysteine and 291 non-alcoholic fatty liver disease in euglycemic patients. Med Sci Monit 22:4146-4151.

292 Inamoto N, Katsuya T, Kokubo Y, Mannami T, Asai T, Baba S, Ogata J, Tomoike H, Ogihara T. 293 2003. Association of methylenetetrahydrofolate reductase gene polymorphism with carotid 294 atherosclerosis depending on smoking status in a Japanese general population. Stroke $29534(7): 1628-1633$.

296 Jeon SB, Kang DW, Kim JS, Kwon SU. 2014. Homocysteine, small-vessel disease, and 297 atherosclerosis: an MRI study of 825 stroke patients. Neurology 83(8):695-701.

298 DOI:10.1212/WNL.0000000000000720.

299 Ji Y, Song B, Xu Y, Fang H, Wu J, Sun S, Zhao L, Shi C, Gao Y, Tao Y, Li Y. 2015. Prognostic 300 significance of homocysteine levels in acute ischemic stroke: a prospective cohort study. Curr 301 Neurovasc Res 12(4):334-340.

302 Jadavji NM, Wieske F, Dirnagl U, Winter C. 2015. Methylen etetrahydrofolate reductase defi 303 ciency alters levels of glutamate and gamma-amin obutyric acid in brain tissue. Mol Genet 304 Metab Rep 3:1-4. DOI: 10.1016/j.ymgmr.2015.02.001. 
305 306 307

Joutel A, Chabriat H. 2017. Pathogenesis of white matter changes in cerebral small vessel diseases: beyond vessel-intrinsic mechanisms. Clin Sci (Lond) 131:635-651.

DOI:10.1042/CS20160380.

Kang SS, Passen EL, Ruggie N, Wong PW, Sora H. 1993. Thermolabile defect of methylenetetrahydrofolate reductase in coronary artery disease. Circulation 88(4 Pt 1):14631469.

Kloppenborg RP, Nederkoorn PJ, van der Graaf Y, Geerlings MI. 2011. Homocysteine and cerebral small vessel disease in patients with symptomatic atherosclerotic disease. The SMARTMR study. Atherosclerosis 216(2):461-466. DOI: 10.1016/j.atherosclerosis.2011.02.027.

Li A, Shi Y, Xu L, Zhang Y, Zhao H, Li Q, Zhao X, Cao X, Zheng H, He Y. 2017. A possible synergistic effect of MTHFR C677T polymorphism on homocysteine level variations increased risk for ischemic stroke. Medicine (Baltimore) 96(51): e9300. DOI: 10.1097/MD.0000000000009300.

Li Q, Yang Y, Reis C, Tao T, Li W, Li X, Zhang JH. 2018. Cerebral small vessel disease. Cell Transplant 27(12):1711-1722. DOI: 10.1177/0963689718795148.

Lu SS, Xie J, Su CQ, Ge S, Shi HB, Hong XN. 2018. Plasma homocysteine levels and intracranial plaque characteristics: association and clinical relevance in ischemic stroke. $B M C$ Neurol 18(1):200. DOI: 10.1186/s12883-018-1203-4. 
323 Li Y, Li M, Yang L, Qin W, Yang S, Yuan J, Jiang T, Hu W. 2019. The relationship between

324 blood-brain barrier permeability and enlarged perivascular spaces: a cross-sectional study. Clin

325 Interv Aging 14:871-878. DOI: 10.2147/CIA.S204269.

326 Nam KW, Kwon HM, Jeong HY, Park JH, Kwon H, Jeong SM. 2019. Serum homocysteine level

327 is related to cerebral small vessel disease in a healthy population. Neurology 92(4):e317-e325.

328 DOI: 10.1212/WNL.0000000000006816.

329 Ou W, Liu X, Shen Y, Li J, He L, Yuan Y, Tan X, Liu L, Zhao J, Wang X. 2014. Association of

330 CVD candidate gene polymorphisms with ischemic stroke and cerebral hemorrhage in Chinese

331 individuals. PLoS One 9(8): e105516. DOI: 10.1371/journal.pone.0105516.

332 Perry IJ, Refsum H, Morris RW, Ebrahim SB, Ueland PM, Shaper AG. 1995. Prospective study

333 of serum total homocysteine concentration and risk of stroke in middle-aged British men. Lancet

$334346(8987): 1395-1398$.

335 Petty GW, Brown RD Jr, Whisnant JP, Sicks JD, O'Fallon WM, Wiebers DO. 2000. Ischemic

336 stroke subtypes: a population-based study of functional outcome, survival, and recurrence. Stroke

337 31(5):1062-1068.

338 Pantoni L. 2010. Cerebral small vessel disease: from pathogenesis and clinical characteristics to

339 therapeutic challenges. Lancet Neurol 9(7):689-701. DOI: 10.1016/S1474-4422(10)70104-6.

340 Pavlovic AM, Pekmezovic T, Obrenovic R, Novakovic I, Tomic G, Mijajlovic M, Sternic N.

341 2011. Increased total homocysteine level is associated with clinical status and severity of white 
342 matter changes in symptomatic patients with subcortical small vessel disease. Clin Neurol

343 Neurosurg 113(9):711-715. DOI: 10.1016/j.clineuro.2011.07.004.

344 Pogliani L, Cerini C, Penagini F, Duca P, Mameli C, Zuccotti GV. 2015. Cerebral ultrasound

345 abnormalities in offsprings of women with C677T homozygous mutation in the MTHFR gene: a

346 prospective study. World J Pediatr 11(2):134-140. DOI: 10.1007/s12519-014-0490-0.

347 Polyzos SA, Kountouras J, Anastasilakis AD, Margouta A, Mantzoros CS. 2015. Association

348 between circulating irisin and homocysteine in patients with nonalcoholic fatty liver disease.

349 Endocrine 49(2):560-562. DOI: 10.1007/s12020-014-0473-x.

350 Piao X, Wu G, Yang P, Shen J, De A, Wu J, Qu Q. 2018. Association between homocysteine and 351 cerebral small vessel disease: A meta-analysis. J Stroke Cerebrovasc Dis 27(9):2423-2430. DOI:

352 10.1016/j.jstrokecerebrovasdis.2018.04.035.

353 Qin X, Li J, Cui Y, Liu Z, Zhao Z, Ge J, Guan D, Hu J, Wang Y, Zhang F, Xu X, Wang X, Xu X, 354 Huo Y. 2012. MTHFR C677T and MTR A2756G polymorphisms and the homocysteine 355 lowering efficacy of different doses of folic acid in hypertensive Chinese adults. Nutr J 11:2.

356 DOI: $10.1186 / 1475-2891-11-2$.

China: a randomized clinical trial. Int J Cancer 141(4):837-847. DOI: 10.1002/ijc.30094. 
361 leukemia risk: a HuGE minireview. Am J Epidemiol 157(7):571-582.

362 Rutten-Jacobs LC, Traylor M, Adib-Samii P, Thijs V, Sudlow C, Rothwell PM, Boncoraglio G,

363 Dichgans M, Meschia J, Maguire J, Levi C, Rost NS, Rosand J, Hassan A, Bevan S, Markus HS.

364 2016. Association of MTHFR C677T Genotype With Ischemic Stroke Is Confined to Cerebral

365 Small Vessel Disease Subtype. Stroke 47(3):646-651. DOI: 10.1161/STROKEAHA.115.011545.

366 Sacco RL, Anand K, Lee HS, Boden-Albala B, Stabler S, Allen R, Paik MC. 2004.

367 Homocysteine and the risk of ischemic stroke in a triethnic cohort: The Northern Manhattan

368 study. Stroke 35(10):2263-2269.

369 Solé X, Guinó E, Valls J, Iniesta R, Moreno V. 2006. SNPStats: a web tool for the analysis of

370 association studies. Bioinformatics 22(15):1928-1929.

371 Shi Y, Wardlaw JM. 2016. Update on cerebral small vessel disease: a dynamic whole-brain

372 disease. Stroke Vasc Neurol 1(3):83-92. DOI: 10.1136/svn-2016-000035.

373 Tan R, Traylor M, Rutten-Jacobs L, Markus H. 2017. New insights into mechanisms of small

374 vessel disease stroke from genetics. Clin Sci (Lond) 131(7):515-531. DOI: 10.1042/CS20160825.

375 Ulvik A, Ueland PM, Fredriksen A, Meyer K, Vollset SE, Hoff G, Schneede J. 2007. Functional

376 inference of the methylenetetrahydrofolate reductase $677 \mathrm{C}>\mathrm{T}$ and $1298 \mathrm{~A}>\mathrm{C}$ polymorphisms

377 from a large-scale epidemiological study. Hum Genet 121(1):57-64.

378 Wardlaw JM, Smith C, Dichgans M. 2013. Mechanisms of sporadic cerebral small vessel disease:

379 insights from neuroimaging. Lancet Neurol 12(5):483-497. DOI: 10.1016/S1474-

Peer] reviewing PDF | (2019:08:40599:2:0:NEW 15 Jan 2020) 
380

381

382

383

384

385

386

387

388

4422(13)70060-7.

Wardlaw JM, Smith EE, Biessels GJ, Cordonnier C, Fazekas F, Frayne R, Lindley RI, O'Brien

JT, Barkhof F, Benavente OR, Black SE, Brayne C, Breteler M, Chabriat H, Decarli C, de

Leeuw FE, Doubal F, Duering M, Fox NC, Greenberg S, Hachinski V, Kilimann I, Mok V,

Oostenbrugge Rv, Pantoni L, Speck O, Stephan BC, Teipel S, Viswanathan A, Werring D, Chen

C, Smith C, van Buchem M, Norrving B, Gorelick PB, Dichgans M; STandards for ReportIng

Vascular changes on nEuroimaging (STRIVE v1). 2013. Neuroimaging standards for research

into small vessel disease and its contribution to ageing and neurodegeneration. Lancet Neurol 12(8):822-838. DOI: 10.1016/S1474-4422(13)70124-8.

Wu W, Guan Y, Xu K, Fu XJ, Lei XF, Lei LJ, Zhang ZQ, Cheng Y, Li YQ. 2016. Plasma homocysteine levels predict the risk of acute cerebral infarction in patients with carotid artery lesions. Mol Neurobiol 53(4):2510-2517. DOI: 10.1007/s12035-015-9226-y.

Wang X, Zhou Y, Zhang M, Wang Y, Qin B. 2018. The methylenetetrahydrofolate reductase genotype 677CT and non-alcoholic fatty liver disease have a synergistic effect on the increasing homocysteine levels in subjects from Chongqing, China. Genes Dis 6(1):88-95. DOI: 10.1016/j.gendis.2018.07.003.

Zee RY, Mora S, Cheng S, Erlich HA, Lindpaintner K, Rifai N, Buring JE, Ridker PM. 2007. Homocysteine, 5,10-methylenetetrahydrofolate reductase $677 \mathrm{C}>\mathrm{T}$ polymorphism, nutrient intake, and incident cardiovascular disease in 24,968 initially healthy women. Clin Chem 53(5):845851. 


\section{Table $\mathbf{1}$ (on next page)}

Univariate analysis results of demographic data, vascular risk factors and laboratory indexes

BMI: Body mass index, TG: Triacylglycerol, LDL-C: Low density lipoprotein cholesterol, HDL-C:

High density lipoprotein cholesterol, SBP: Systolic blood pressure, DBP: Diastolic blood pressure, FBG: Fasting blood glucose. 
1 Table 1 Univariate analysis results of demographic data, vascular risk factors and

2 laboratory indexes

\begin{tabular}{|c|c|c|c|c|c|}
\hline & & $\begin{array}{l}\text { Case group } \\
\qquad(n=163)\end{array}$ & $\begin{array}{l}\text { Control group } \\
\qquad(n=326)\end{array}$ & $\chi^{2 / t}$ & $P$ \\
\hline Age (Years) & & $63.42 \pm 7.13$ & $63.21 \pm 6.98$ & 0.309 & 0.804 \\
\hline BMI $\left(\mathrm{Kg} / \mathrm{m}^{2}\right)$ & & $22.18 \pm 4.61$ & $22.37 \pm 4.95$ & 0.419 & 0.716 \\
\hline & $<10000$ & $18(11.04 \%)$ & $39(11.96 \%)$ & & \\
\hline & $10000-20000$ & $48(29.45 \%)$ & $92(28.22 \%)$ & & \\
\hline & $>20000$ & $97(59.51 \%)$ & $195(59.82 \%)$ & 0.138 & 0.933 \\
\hline & Primary school and & & & & \\
\hline & & $69(42.33 \%)$ & $130(39.88 \%)$ & & \\
\hline
\end{tabular}

Educational level Junior high school 58(35.58\%) $\quad 114(34.97 \%)$

Senior high school

$$
36(22.09 \%) \quad 82(25.15 \%)
$$

and above

$0.596 \quad 0.742$

Farmer $\quad 71(43.56 \%) \quad 131(40.18 \%)$

Occupation

$\begin{array}{lllll}\text { Worker } \quad 61(37.42 \%) & 123(37.73 \%) & 0.788 & 0.674\end{array}$


Civil

$\begin{array}{ccc} & 31(19.02 \%) & 72(22.09 \%) \\ \text { servant/teacher/doctor } & & \\ \text { Yes } & 40(24.54 \%) & 74(22.70 \%)\end{array}$

Drinking

No

$123(75.46 \%)$

$252(77.30 \%)$

0.206

0.650

Yes

$48(29.45 \%)$

$73(22.39 \%)$

Hyperlipidaemia

No $\quad 115(70.55 \%) \quad 253(77.61 \%) \quad 2.905 \quad 0.088$

Total cholesterol

$(\mathrm{mmol} / \mathrm{L})$

$4.45 \pm 1.53$

$4.30 \pm 1.41$

$(\mathrm{m} m \mathrm{ml} / \mathrm{L})$

$\mathrm{TG}(\mathrm{mmol} / \mathrm{L})$

$1.54 \pm 1.22$

$1.42 \pm 1.09$

$1.062 \quad 0.311$

LDL-C (mmol/L)

$2.62 \pm 1.34$

$2.39 \pm 1.37$

$1.776 \quad 0.073$

HDL-C (mmol/L)

$1.24 \pm 0.48$

$1.33 \pm 0.56$

$1.847 \quad 0.068$

Yes

$54(33.13 \%) \quad 66(20.25 \%)$

Hypertension

\begin{tabular}{|c|c|c|c|}
\hline No & $109(66.87 \%)$ & $260(79.75 \%)$ & 9.740 \\
\hline Yes & $50(30.67 \%)$ & $64(19.63 \%)$ & \\
\hline
\end{tabular}

Diabetes

No

$116(69.33 \%) \quad 262(80.37 \%)$

$6.797 \quad 0.009$ 


$$
\text { Yes } \quad 49(30.06 \%) \quad 65(19.94 \%)
$$

Smoking

No

$\begin{array}{llll}114(69.94 \%) & 261(80.06 \%) & 6.228 & 0.013\end{array}$

SBP $(\mathrm{mmHg})$

$144.83 \pm 13.92$

$138.78 \pm 13.88$

$4.535<0.001$

DBP (mmHg)

$90.74 \pm 9.08$

$86.96 \pm 8.64$

$4.41<0.001$

FBG $(\mathrm{mmol} / \mathrm{L})$

$6.62 \pm 2.85$

$4.063<0.001$

3 BMI: Body mass index, TG: Triacylglycerol, LDL-C: Low density lipoprotein cholesterol, HDL-

4 C: High density lipoprotein cholesterol, SBP: Systolic blood pressure, DBP: Diastolic blood 5 pressure, FBG: Fasting blood glucose.

6 
Table 2 (on next page)

Univariate analysis results of allele and genotype frequency

HWE: Hardy-Weinberg equilibrium. 
1 Table 2 Univariate analysis results of allele and genotype frequency

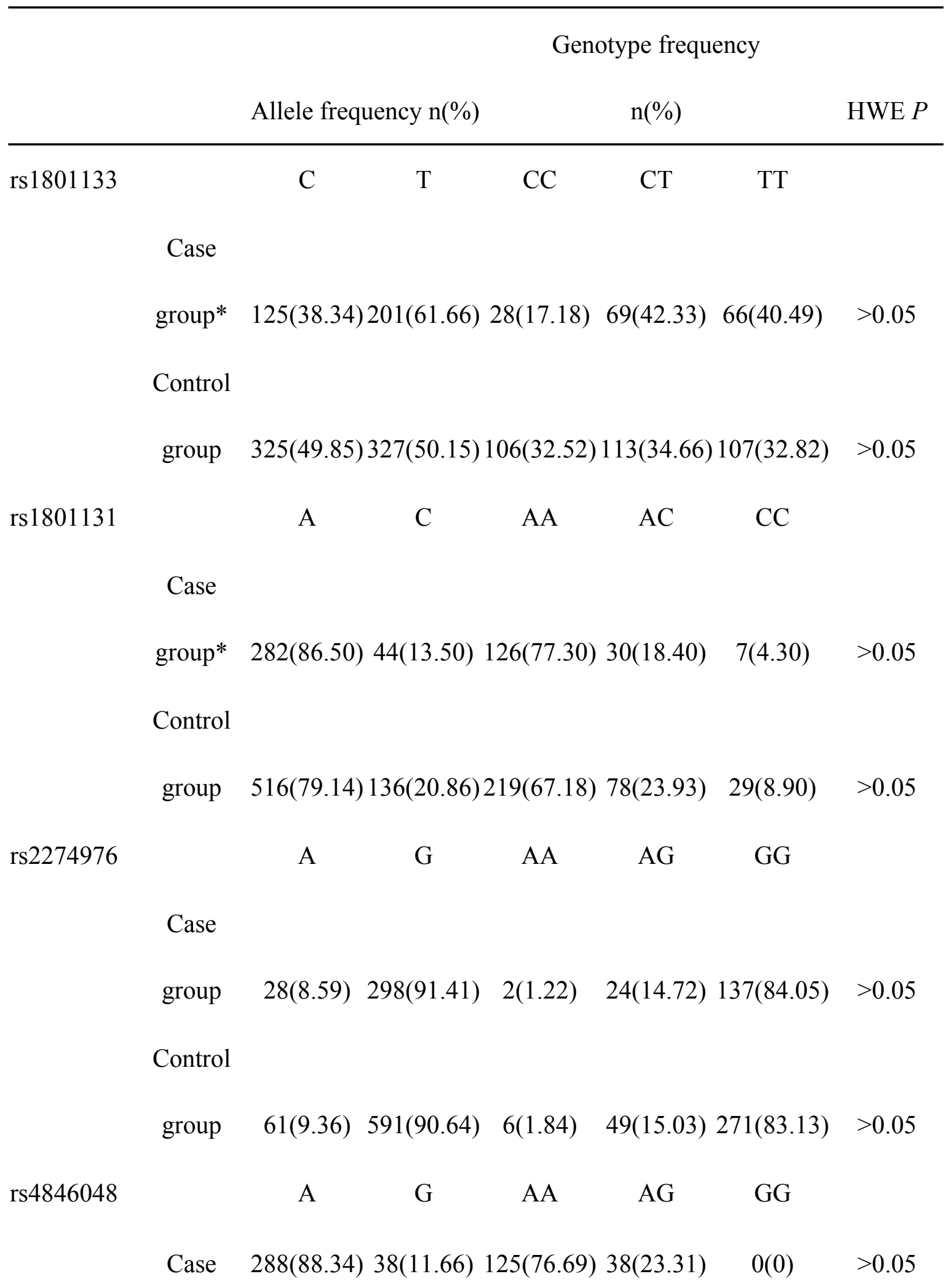




\begin{tabular}{|c|c|c|c|c|c|c|c|}
\hline & Control & & & & & & \\
\hline & group & $581(89.11)$ & $71(10.89)$ & $255(78.22)$ & $71(21.78)$ & $0(0)$ & $>0.05$ \\
\hline rs4846049 & & $\mathrm{G}$ & $\mathrm{T}$ & GG & GT & TT & \\
\hline
\end{tabular}

Case

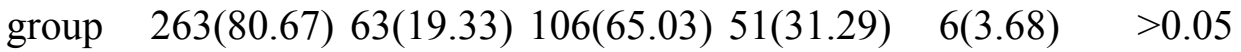

Control

$\begin{array}{lllll}\text { group } & 525(80.52) 127(19.48) 213(65.34) 99(30.37) & 14(4.29) & >0.05\end{array}$

rs 13306561

$\mathrm{C} \quad \mathrm{T} \quad \mathrm{CC}$

CT

TT

Case

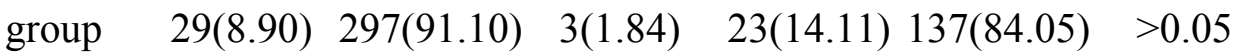

Control

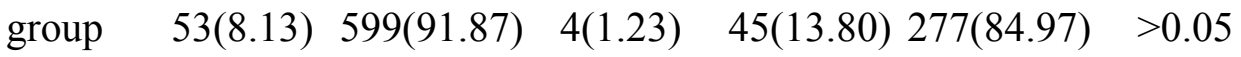

rs3737964

A

G

AA

AG $\quad$ GG

Case

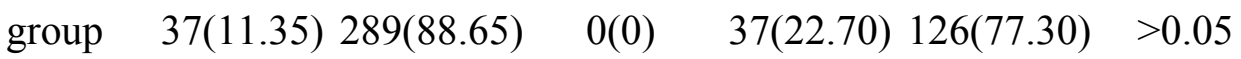

Control

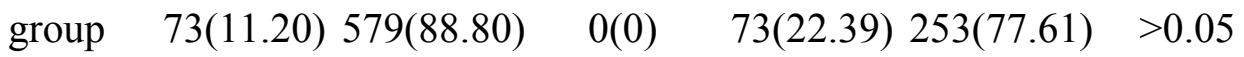

$2 *: P<0.05$, vs allele frequency and genotype frequency of the control group.

3 HWE: Hardy-Weinberg equilibrium. 


\section{Table 3 (on next page)}

Independent association between different genotypes of the MTHFR rs1801133 and rs1801131 and CSVD

MTHFR: Methylenetetrahydrofolate reductase, CSVD: Cerebral small vessel disease, OR: odds ratio, $\mathrm{Cl}$ : confidence interval. 
1 Table 3 Independent association between different genotypes of the MTHFR rs1801133 and 2 rs 1801131 and CSVD

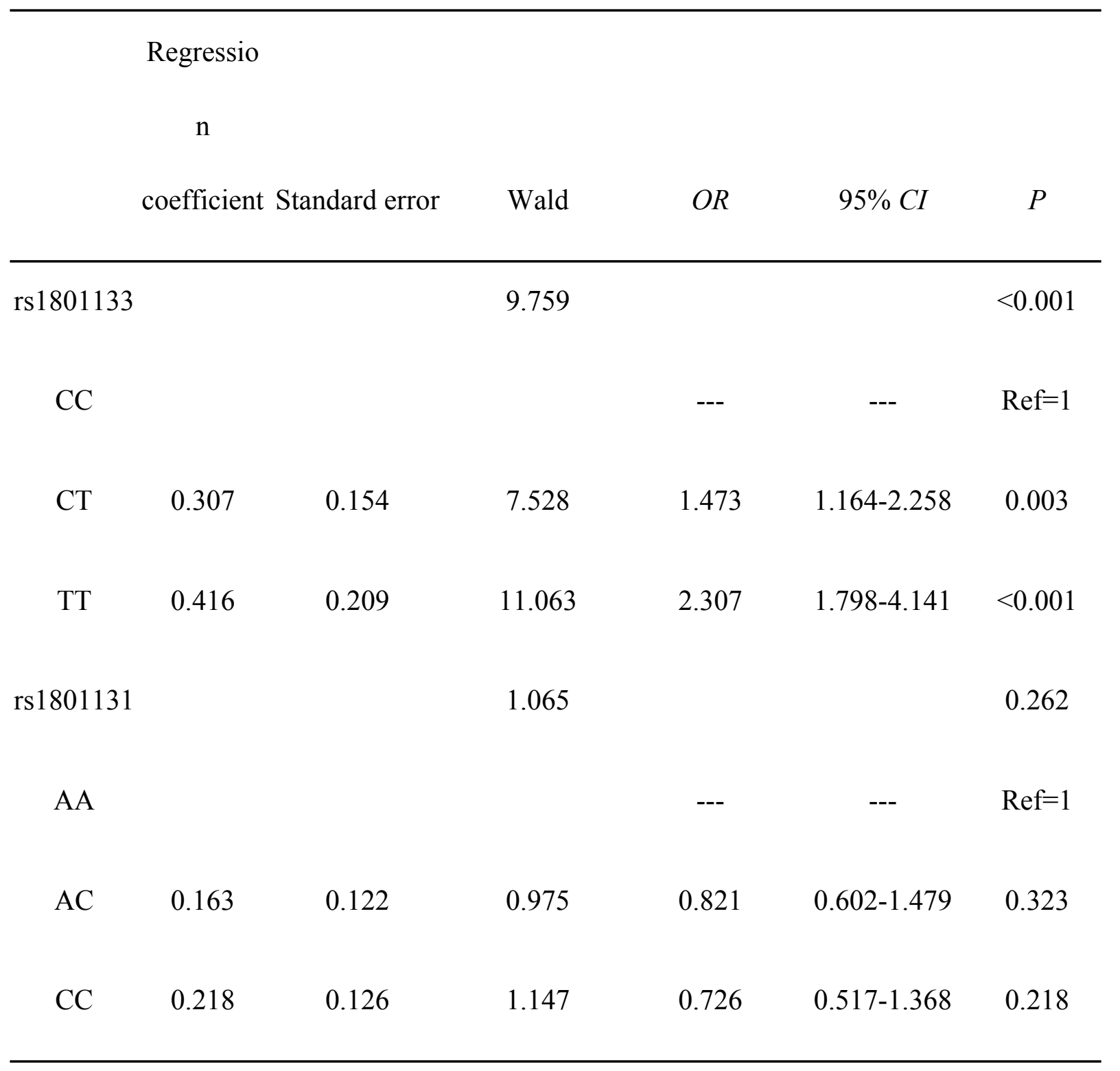

3 MTHFR: Methylenetetrahydrofolate reductase, CSVD: Cerebral small vessel disease, OR: odds 4 ratio, $C I$ : confidence interval. 


\section{Table 4 (on next page)}

Plasma Hcy levels in the MTHFR rs1801133 CC, CT and TT genotype

*: $P<0.05$, vs CC genotype; ${ }^{\diamond}: P<0.05$, vs CT genotype. 
1 Table 4 Plasma Hcy levels in the MTHFR rs 1801133 CC, CT and TT genotype

\begin{tabular}{ccc}
\hline & $\mathrm{n}$ & Plasma Hcy levels $(\mathrm{pg} / \mathrm{ml})$ \\
\hline CC genotype & 134 & $14.96 \pm 4.85$ \\
CT genotype & 182 & $17.04 \pm 5.68^{*}$ \\
TT genotype & 173 & $19.91 \pm 8.73^{* \diamond}$ \\
$F$ & & 9.094 \\
$P$ & & $<0.001$ \\
\hline
\end{tabular}

$2 *: P<0.05$, vs CC genotype; $\diamond: P<0.05$, vs CT genotype. 


\section{Table 5 (on next page)}

Frequencies of the six common combination genotypes 
1 Table 5 Frequencies of the six common combination genotypes

\begin{tabular}{ccccccc}
\hline \multicolumn{7}{c}{ Six common genotype combinations $\mathrm{n}(\%)$} \\
& TTAA & CTAA & CTAC & CCAC & CCAA & CCCC \\
\hline Case group & $66(40.49)$ & $47(28.83)$ & $22(13.50)$ & $6(3.68)$ & $18(11.04)$ & $4(2.45)$ \\
Control & & & & & & \\
group & $106(32.52)$ & $72(22.09)$ & $40(12.27)$ & $49(15.03)$ & $26(7.98)$ & $31(9.51)$ \\
$\chi^{2}$ & & & & & & \\
$P$ & & & & & & \\
\hline
\end{tabular}

2 
Table 6(on next page)

Plasma Hcy levels of the six common combination genotypes 
1 Table 6 Plasma Hcy levels of the six common combination genotypes

\begin{tabular}{ccc}
\hline & $\mathrm{n}$ & \\
& 172 & Plasma Hcy levels $(\mathrm{pg} / \mathrm{ml})$ \\
\hline TTAA & 119 & $19.93 \pm 8.71$ \\
CTAA & 62 & $17.76 \pm 5.72 *$ \\
CTAC & 55 & $16.32 \pm 5.28^{*}$ \\
CCAC & 44 & $14.75 \pm 4.64 * \diamond$ \\
CCAA & 35 & $15.94 \pm 4.93 * \diamond$ \\
CCCC & & $14.06 \pm 4.49 * \diamond \square$ \\
$P$ & & 6.275 \\
\hline
\end{tabular}

$2 \quad *: P<0.05$, vs TTAA genotype; $\diamond: P<0.05$, vs CTAA genotype; $\square: P<0.05$, vs CTAC genotype 